Hydrology and Earth System Sciences, 10, 19-29, 2006

www.copernicus.org/EGU/hess/hess/10/19/

SRef-ID: 1607-7938/hess/2006-10-19

European Geosciences Union

\title{
Transport at basin scales: 1. Theoretical framework
}

\author{
A. Rinaldo, G. Botter, E. Bertuzzo, A. Uccelli, T. Settin, and M. Marani \\ International Centre for Hydrology "Dino Tonini” and Dipartimento IMAGE, Università di Padova, via Loredan 20, 35131 \\ Padova, Italy
}

Received: 6 July 2005 - Published in Hydrology and Earth System Sciences Discussions: 23 August 2005

Revised: 22 November 2005 - Accepted: 1 December 2005 - Published: 8 February 2006

\begin{abstract}
The paper describes the theoretical framework for a class of general continuous models of the hydrologic response including both flow and transport of reactive solutes. The approach orders theoretical results appeared in disparate fields into a coherent theoretical framework for both hydrologic flow and transport. In this paper we focus on the Lagrangian description of the carrier hydrologic runoff and of the processes embedding catchment-scale generation and transport of matter carried by runoff. The former defines travel time distributions, while the latter defines lifetime distributions, here thought of as contact times between mobile and immobile phases. Contact times are assumed to control mass transfer in a well-mixed approximation, appropriate in cases, like in basin-scale transport phenomena, where the characteristic size of the injection areas is much larger than that of heterogeneous features. As a result, we define general mass-response functions of catchments which extend to transport of matter geomorphologic theories of the hydrologic response. A set of examples is provided to clarify the theoretical results towards a computational framework for generalized applications, described in a companion paper.
\end{abstract}

\section{Introduction}

The effective management of hydrological systems, including e.g. the design of hydraulic structures, of the general architecture of systems capable of mitigating the effects of floods and droughts and of measures aimed at improving the quality of receiving water bodies, can benefit from the use of reliable models describing hydrological fluxes and storage terms both in space and time (e.g. Beven and Freer, 2001; Maurer and Lettenmaier, 2003). New tools and open problems for models of the hydrologic response have been re-

Correspondence to: A. Rinaldo

(andrea.rinaldo@unipd.it) cently summarized by Montanari and Uhlenbrook (2004), and yet analogs for general transport processes are lagging behind, especially if solidly rooted in the stochastic framework that seems appropriate for large-scale applications. General transport models would serve well, however, both research and applications, given the timeliness of design criteria that include directly concepts of probability. Management objectives require, in fact, models capable of i) reproducing system functioning as described by observations; and ii) predicting system functioning under conditions and during events which have not been observed, possibly generating statistical ensembles of events. This must be possible without the burden of making unphysical or unrealistic assumptions, like, typically, statistical stationarity of the response of manned and ever-changing watersheds. Thus one can hardly overestimate the importance of basin-scale models of transport for society at large.

The formulation of transport by travel time distributions serves well the above scopes (Rodriguez-Iturbe and Valdes, 1979; Gupta et al., 1980; Dagan, 1989; Rinaldo and Rodriguez-Iturbe, 1996). We shall address here this formulation in a framework somewhat broader and more comprehensive than that of the original approach. In fact, here we collect independent results from transport theories to propose a formulation that applies regardless of whether we deal with flow or with transport models at catchment scales (e.g. as in Rinaldo and Marani, 1987; Rinaldo et al., 1989, 1991; Rinaldo and Rodriguez-Iturbe, 1996; Cvetkovic and Dagan, 1996; Gupta and Cvetkovic, 2002; Destouni and Graham, 1995; Botter and Rinaldo, 2003; Botter et al., 2005) aimed at the large-scale collection and objective manipulation of geomorphic, hydrologic or land use data.

This paper is organized as follows. An introductory framework reviews the kinematics and the elements of general transport theory that allow us to blend flow and transport of matter for a single transport volume. The ensuing sections use the theoretical results obtained for a single transport

(C) 2006 Author(s). This work is licensed under a Creative Commons License. 
volume to obtain a formulation valid for arbitrary sequences (in series or in parallel) of transport states, distinguishing the effective functioning of any geomorphic paths upon the fraction of input rainfall conveyed therein. A conclusive section proposes a few examples aimed at clarifying a somewhat convolute procedure, which is related to the naturally nested structure of control volumes within a catchment rather than to unnecessary complications of our models.

\section{Theoretical framework}

\subsection{Flow}

Once net rainfall is suitably partitioned into surface and subsurface pathways, the flux of the water carrier within natural formations is seen as a conservative process where water particles move within the control volume towards the outlet without significant variations of their mass. Let thus $m_{w}$ be the (time-independent) water mass transported by a single particle injected at time $t_{0}=0$ in the initial position $\mathbf{x}_{0}$. Each trajectory is defined by its Lagrangian coordinate $\mathbf{X}(t)=\mathbf{x}_{0}+\int_{0}^{t} \mathbf{v}(\mathbf{X}(\tau), \tau) d \tau$, where $\mathbf{v}(\mathbf{x}, t)$ is the point value of the velocity vector. The spatial distribution of water concentration in the transport volume $\mathcal{V}$ as a result of the injection of a single particle is given by Taylor (1921):

$c_{w}(\mathbf{x}, t) \propto m_{w} \delta(\mathbf{x}-\mathbf{X}(t))$,

where $\delta(\cdot)$ is Dirac's delta distribution and, without loss of generality, we have assumed unit porosity within the whole control volume (i.e. $\int_{\mathcal{V}} c_{w} d \mathbf{x}=m_{w}$ ). Note that the proportionality in Eq. (1) stems from the assumption of costant porosity of the transport volume along the flow paths, which proves feasible for a variety of case of interest (Dagan, 1989). Equation (1) states that, in the one-particle one-realization case, volumetric water concentration (water mass per unit transport volume) is nonzero only at the site where the particle is instantaneously residing (i.e. at its trajectory). Thus uncertainty in the dynamical specification of the particle (i.e. the evolution in time and space of the trajectory $\mathbf{X}(t)$ of the labeled, traveling "water particle") is reflected in the transport process.

Owing to the heterogeneity which characterizes transport processes and environments at basin scale, the trajectory is seen as a random function. Let therefore $g(\mathbf{X}) d \mathbf{X}$ be the probability that the particle is found within the infinitesimal volume $d \mathbf{X}$ located around the position $\mathbf{X}$ at time $t$ (notice that the functional dependence $g(\mathbf{X})$ implies $g(\mathbf{x}, t)$ in terms of cartesian coordinates because of the evolution of the trajectory with time). The ensemble average concentration $\left\langle c_{w}(\mathbf{x}, t)\right\rangle$ is given by the classic relation (Taylor, 1921; Dagan, 1989):

$\left\langle c_{w}(\mathbf{x}, t)\right\rangle=\int_{-\infty}^{\infty} m_{w} \delta(\mathbf{x}-\mathbf{X}) g(\mathbf{X}) d \mathbf{X}=m_{w} g(\mathbf{x}, t)$
The distribution $g(\mathbf{x}, t)$ is usually called displacement probability density function. Important models describing displacement distributions, $g$, or $\left\langle c_{w}\right\rangle$ (from Eq. $2 g \propto\left\langle c_{w}\right\rangle$ ), notably the cases deriving from the Fokker-Planck equation, are reported in the literature (see, for a summary relevant to hydrology, Rinaldo et al., 1991). Note that the above theoretical link between displacement distributions and mean concentrations allows the equivalence of the rate of change of displacement covariances (heuristically, the moments of inertia of the displaced particles) with half the dispersion coefficient of the Eulerian problem, originating the definition of shear-flow, hydrodynamic or geomorphologic dispersion. Details on the nature of the dispersion tensor can be found elsewhere (e.g. Dagan, 1989).

The displacement pdf $g(\mathbf{x}, t)$ due to the kinematics of the carrier flow determines the travel time distribution $f(t)$ of the water carrier within the control volume. The definition of the undergoing travel time distribution is related to the possibility of identifying a suitable control section for the transport process considered. We thus assume that the time $t$ at which a particle crosses the control section is unique and, most importantly, that all particles injected in $\mathcal{V}$ ensuing from $\mathbf{x}_{0} \in \mathcal{V}$ must transit the predefined control-section. The probability density of travel times is proportional to the instantaneous mass flux at the absorbing barrier of the control volume (Dagan, 1989). In fact water mass in storage within the control volume $M_{w}(t)$ is expressed by:

$$
\begin{aligned}
M_{w}(t) & =\int_{\mathcal{V}}<c_{w}>d \mathbf{x}=m_{w} \int_{\mathcal{V}} g(\mathbf{x}, t) d \mathbf{x}= \\
& =m_{w} P(T \geq t)
\end{aligned}
$$

where $P(T \geq t)$ is the probability that the residence time is larger than current time $t$. Thus, by continuity, one has $d M_{w}(t) / d t=I-Q_{w}$ (where $I[M][T]^{-1}$ is the mass water input and $Q_{w}(t)[M][T]^{-1}$ is the mass flux at the outlet of $\mathcal{V}$ ), and therefore, for an instantaneous water pulse (i.e. $\left.I(t)=m_{w} \delta(t)\right)$ :

$Q_{w}(t)=-m_{w} \frac{d P(T \geq t)}{d t}=m_{w} f(t)$ for $t>0$

where $f(t)$ is the probability density function (pdf) of travel times for the water carrier. In surface hydrology, when the input is a unit of net rainfall, such pdf is usually termed the instantaneous unit hydrograph.

In using the travel time formulation of transport in surface hydrology, two courses have been pursued: one course assumes the form of the pdf, and characterizes it by some parameters of clear physical meaning like mean travel times. An example of this are the exponential pdf's used to describe travel times of water particles in the original approach by Rodriguez-Iturbe and Valdes (1979) to derive the geomorphologic unit hydrograph. The second course exploits the equivalence of water fluxes and pdf's to deduce travel times from the equations of motion. Eulerian, Lagrangian or travel time approaches therefore may differ formally although they 
are derived from the same assumptions. The common prejudice of considering one approach in principle superior to the other is therefore incorrect. A discussion on the relative balance of merits of the above approaches can be found in Dagan (1989).

\subsection{Transport}

We now turn to reactive transport of solutes carried by hydrologic waters in the same framework depicted in Sect. 2.1. A given amount of solute (of mass $m_{s}$ ) is injected within the control volume through an instantaneous release of water, and is thus allowed to move within the transport volume driven by the hydrologic carrier flow and to exchange mass with the surrounding environment. The "reactive" character of the transport is described by the (spatial and/or temporal) variability of the solute mass associated with the water particles moving within the control volume, that is, the function $m_{s}=m_{s}\left(\mathbf{X}, t ; t_{0}\right)$ which embeds physical, chemical or biological exchanges with immobile phases in some contact with the carrier flow.

One-particle, one-realization concentration fields resulting from the injection of a single reactive particle are given by the following equation:

$c_{s}\left(\mathbf{x}, t ; \mathbf{x}_{0}, t_{0}\right) \propto m_{s}\left(\mathbf{X}, t ; t_{0}\right) \delta(\mathbf{x}-\mathbf{X}(t))$,

The reactive components involved define the instantaneous solute mass $m_{s}$ attached to the moving particle without affecting the trajectory $\mathbf{X}$ of the particle itself which is determined by the usual kinematic relationship. The mass transfer occurring between the carrier and immobile phases (e.g. chemical or physical sorption, ion exchange, precipitation) leads in general to variability for $m$ both in time and space. We assume, however, that the injection area is much larger than any correlation scale of heterogeneous transport properties and/or that the temporal scales relevant for the undergoing advective processes are smaller than (or, at most, comparable with) the characteristic times for the reaction processes. This suggests (Rinaldo et al., 1989; Rinaldo and RodriguezIturbe, 1996; Botter et al., 2005) that the spatial gradients of mass exchange become negligible and that, therefore, the contact times drive mass transfer between phases (i.e. the well-mixed approximation). The injection of identical particles labeled by carrier and solute masses $m_{w}, m_{s}$ at different initial locations $\mathbf{x}_{\mathbf{0}}$ at time $t_{0}$ produces, at time $t>t_{0}$, the sampling of different trajectories $\mathbf{X}(t)$ but yields roughly the same temporal evolution of the mass of solute transported $m_{s}\left(t-t_{0}, t_{0}\right)$, which thus depends (for a given injection time $t_{0}$ ) solely on the time available for the reaction processes, $t-t_{0}$. The expected value of the volumetric concentration $\left\langle c_{s}(\mathbf{x}, t)\right\rangle$ (solute mass for unit transport volume) is then given, from Eq. (3), by the relation (Rinaldo and RodriguezIturbe, 1996):

$$
\left\langle c_{s}\left(\mathbf{x}, t ; t_{0}\right)\right\rangle=m_{s}\left(t-t_{0}, t_{0}\right) g\left(\mathbf{x}, t-t_{0}\right)
$$

where the similarity of structure with respect to passive transport stems from the fact that $m_{s}$ is unaffected by ensemble averaging. Thus we obtain a generalization of Taylor's theorem for reactive transport problems. The displacement distribution $g$ defines the structure of the carrier residence time distribution within the control volume and thus epitomizes the complex chain of events determining the hydrologic flow. The mass function $m_{s}\left(t-t_{0}, t_{0}\right)$ accounts for all physical and chemical processes which determine the temporal variability of the solute mass transported by the moving water particles. The decoupling of the reaction component from the transport problem is quite expedient because the displacement and the travel time distributions derived in the previous section may be employed.

The solute mass instantaneously stored in the water carrier within the transport volume $\mathcal{V}$ (as a result of a solute injection occurring at $t=t_{0}$ ) may be thus expressed by the use of Eq. (6) as:

$$
\begin{aligned}
M_{s}(t) & =\int_{\mathcal{V}}<c_{s}\left(\mathbf{x}, t ; t_{0}\right)>d \mathbf{x} \\
& =m_{s}\left(t-t_{0}, t_{0}\right) P\left(T \geq t-t_{0}\right)
\end{aligned}
$$

where $P(T \geq t)$ is the probability that the residence time is larger than the current time $t$. Thus, deriving Eq. (7) with respect to $t$, one has:

$\frac{d M_{s}(t)}{d t}=-m_{s}\left(t-t_{0}, t_{0}\right) f\left(t-t_{0}\right)+\frac{d m_{s}}{d t} P\left(T \geq t-t_{0}\right)(8)$

where the last term of the right-hand side of the above equation represents the rate of solute, say $R\left([M][T]^{-1}\right)$, transferred from the immobile phase to the water carrier due to the active reaction processes. Since for $t>t_{0}$ by continuity one has $d M_{s} / d t=-Q_{s}+R$ (where $Q_{s}[M][T]^{-1}$ is the solute flux at the outlet of $\mathcal{V}$ ), by comparison with Eq. (8) we obtain:

$Q_{s}\left(t ; t_{0}\right)=m_{s}\left(t-t_{0}, t_{0}\right) f\left(t-t_{0}\right)$ for $t>t_{0}$

Equation (9) expresses the solute flux at the outlet due to the injection within the control volume at $t=t_{0}$ of an instantaneous water pulse carrying a solute mass $m_{s}$ which is timedependent owing to mass exchange processes.

In what follows, we assume that the solutes transported by the carrier undergo sorption phenomena with other immobile phases in contact with the water flow (e.g. soil grains, bed sediment, dead-end zones). The mass transfer between the phases is therefore driven by the difference between the solute concentration sorbed in the immobile phase and the solute concentration, say $C$, characterizing the water particles moving along the control volume (solute mass for unit water volume) (van Genuchten, 1981). The latter may be straightforwardly derived by use of Eqs. (2) and (6) as:

$C\left(t-t_{0}, t_{0}\right)=\rho \frac{\left\langle c_{s}\left(\mathbf{x}, t ; t_{0}\right)\right\rangle}{\left\langle c_{w}\left(\mathbf{x}, t ; t_{0}\right)\right\rangle}=\rho \frac{m_{s}\left(t-t_{0}, t_{0}\right)}{m_{w}}$ 
where $\rho$ is the (constant) water density $\left([M][L]^{-3}\right)$. Notice that in Eq. (10) the capital letter $C$ is employed for the solute concentration of the water particles (solute mass for unit water volume), so as to highlight the difference with respect to the volumetric concentration of solute $c_{s}$ (mass for unit transport volume). Notice that at a given time $t$, the water particles injected into the system at the same injection time $t_{0}$ are all marked by the same resident concentration $C\left(t-t_{0}, t_{0}\right)$, independently from their trajectory. This is, of course, an important assumption which nonetheless seems applicable to most cases where rainfall is the driving factor (Botter et al., 2005).

Note that it is appropriate to state clearly the mathematical analogies that stem from the relation

$\tau=t-t_{0}$

where $\tau$ is the travel time of a single particle within the control volume after injection at time $t_{0}$, thereby the contact time between phases, and $t$ is chronological time. Thus, one may easily express the solute concentration of the water carrier as a function of only two of the above timescales (e.g. $C=C\left(\tau, t_{0}\right)$, or $C=C(\tau, t)$, see below $)$.

Within the above framework, solute mass transported by the water carrier, $m_{s}$, is thus defined by the rate of change of the scalar property $C\left(t-t_{0}, t_{0}\right)$ attached to the mobile phase. Incidentally, when the scalar is simply the density of the carrier i.e. $C\left(t-t_{0}, t_{0}\right)=$ const $=\rho$, the above derivation reduces to the description of flowrates. In the general case, instead, the temporal variability of the function $C$ (which retains all sorption/desorption processes determining the temporal variability of the mass transported by the moving particles) is related to the active reaction processes between the phases. For the sake of simplicity, linear rate-limited kinetics are assumed to drive the temporal evolution of the concentration function $C\left(t-t_{0}, t_{0}\right)$ (Rinaldo and Marani, 1987):

$$
\frac{\partial C\left(\tau, t_{0}\right)}{\partial \tau}=k\left(\frac{N(t)}{k_{D}}-C\left(\tau, t_{0}\right)\right)
$$

where $N\left(\left[M M^{-1}\right]\right)$ is the concentration in the immobile phase (properly transformed by $k_{D}\left(\left[L^{3} M^{-1}\right]\right)$, the equivalent of a partition coefficient) and $k\left(\left[T^{-1}\right]\right)$ is the overall rate coefficient of the reaction kinetics between mobile and immobile phases. According to the well-mixed assumption, the concentration in the immobile phase $N$ is assumed to solely depend on time and not on the position $\mathbf{x}$. The temporal evolution of the function $N(t)$ may be thus described on the basis of a global (rather than local) mass balance, applicable to each "state" which is physically meaningful to identify. This is not the case, for instance, in the other approaches well known from the literature (Cvetkovic and Dagan, 1994).

An important indicator of the validity of the above assumptions comes from an application where the carrier flow is in steady state, which is a particular case of the above framework for constant input flowrates (Botter et al., 2005). Consider a steady-state flow through a generic heterogeneous medium and assume that the underlying Eulerian velocity field is a stationary random vectorial function $\mathbf{v}(\mathbf{x})$. The ensemble mean of the local velocity $\mathbf{v}$ is assumed to be positive (i.e. a mean flow direction is determined) and - without loss of generality - aligned with one axis. Under the above assumptions, the transport domain may be thought of as a collection of independent and stationary streamlines, which are characterized by different residence times owing to the heterogeneity of the transport properties involved. Solute particles injected within the flow field, or released from the soil, are simultaneously advected by the carrier and affected by sorption-desorption processes with immobile phases in contact with the water flow. In this context, a noteworthy simplification of the transport problem may be achieved by projecting the transport equation along a single streamline and embedding all the heterogeneities of the transport properties within a single variable, the travel time $\tau$ (for details see e.g. Cvetkovic and Dagan, 1994). If we assume that linear and reversible sorption processes occur between the mobile and the immobile phases, mass conservation yields:

$$
\frac{\partial C(\tau, t)}{\partial t}+\frac{\partial C(\tau, t)}{\partial \tau}=R=k_{2} N(\tau, t)-k_{1} C(\tau, t)
$$

and

$$
\frac{\partial N(\tau, t)}{\partial t}=k_{1} C(\tau, t)-k_{2} N(\tau, t)
$$

where $C\left[M L^{-3}\right]$ represents the solute concentration in the mobile phase, $N\left[M L^{-3}\right]$ is the solute concentration in the immobile phase (mass of solute per unit fluid volume), $R$ $\left[M L^{-3} T^{-1}\right]$ is the sink/source term due to chemical and/or physical reactions and $k_{1}, k_{2}\left[T^{-1}\right]$ are the forward and backward reaction coefficients, respectively. It is worth mentioning that $\tau$ is the time needed for a particle injected in $\mathbf{x}_{0}$ at $t=0$ (i.e. $\mathbf{X}(0)=\mathbf{x}_{0}$, with $\mathbf{X}(t)=(X(t), Y(t), Z(t))$ as usual the trajectory of the particle) to reach a control plane, perpendicular to the mean flow direction, located at a distance $x$ (measured along the mean flow direction) from the injection site (Cvetkovic and Dagan, 1994):

$\tau(x)=\int_{0}^{x} \frac{d \xi}{u(\xi, \eta(\xi), \zeta(\xi))}$

The quantities $\eta$ and $\zeta$ in Eq. (15) are the transversal displacements of the considered particle, i.e. $\eta(x)=Y(\tau(x))$ and $\zeta(x)=Z(\tau(x))$ (for a complete treatment, only sketched here, see Cvetkovic and Dagan $(1994,1996))$. It should be noted that Eq. (13) is actually fully three-dimensional, since the Lagrangian variable $\tau$ retains the 3-D structure of the velocity field. Furthermore, in Eq. (13) we neglect pore-scale dispersion; in heterogeneous formations, in fact, pore scale dispersion may only affect the local values of resident concentrations but bears a negligible overall effect on global quantities, such as mass fluxes and the spatial/temporal plume moments (Dagan, 1989), particularly in the case of reactive solutes (see the discussion e.g. in Botter et al., 2005). 
When considering basin scales, it has been shown that ensemble averaging over different injection points $\mathbf{x}_{0}$ embedding source areas larger than the scales characteristic of heterogeneous properties (thereby typically for particles injected by rainfall patterns) smooth out the dependence on the features of the single trajectory and that the above framework forced to steady state often gives negligible differences with respect to the full Lagrangian framework, and that in practice one has $N(t, \tau) \sim N(t)$ (Botter et al., 2005). This leads to the simplified formulation provided by Eq. (12), where the spatial gradients of immobile concentration are neglected (for a detailed discussion, see e.g. Botter et al., 2005).

The solute mass flux [M/T] due to an instantaneous injection of a water flux $J(t)=\left(m_{w} / \rho\right) \delta\left(t-t_{0}\right)\left([L]^{3}[T]^{-1}\right)$ may be thus expressed by the use of Eqs. (9) and (10) as:

$$
\begin{aligned}
Q_{s}\left(t, t_{0}\right) & =\frac{m_{w}}{\rho} C\left(t-t_{0}, t_{0}\right) f\left(t-t_{0}\right) \\
& =J\left(t_{0}\right) \Delta t_{0} C\left(t-t_{0}, t_{0}\right) f\left(t-t_{0}\right)
\end{aligned}
$$

where $J\left(t_{0}\right) \Delta t_{0}=m_{w} / \rho$ is the water volume injected in the system during the time interval $\Delta t_{0}$. Equation (16) states the equality between the mass response function (i.e. the solute release corresponding to a unit water input) and the product between the carrier transfer function $f$ (i.e. the travel time distribution for the water flow) and its solute concentration C.

Flowrates $\left[L^{3} / T\right]$ (constant $m_{w}$ ) and mass fluxes $[M / T]$ (variable $m_{S}$ ) generated by an arbitrary sequence of rainfall volumes $J(t)\left[L^{3} / T\right]$ (which we may treat as clean for $\tau=0$, i.e. $\left.C\left(0, t_{0}\right) \equiv 0\right)$ are thus derived, for a single transport volume, from Eqs. (4) and (16):

$Q_{w}(t)=\int_{0}^{t} d t_{0} J\left(t_{0}\right) f\left(t-t_{0}\right)\left[L^{3} / T\right]$

and

$$
Q_{s}(t)=\int_{0}^{t} d t_{0} J\left(t_{0}\right) C\left(t-t_{0}, t_{0}\right) f\left(t-t_{0}\right)[M / T]
$$

in the two respective cases.

It is important to notice that in the case of unsteady forcing one may also need to distinguish resident concentrations, $C\left(t-t_{0}, t_{0}\right)$, from flux concentrations, say $C^{F}(t)$, at the outlet of single transport volumes (thereby only a function of current time $t$ ):

$C^{F}(t)=\frac{Q_{s}(t)}{Q_{w}(t)}$

$C^{F}(t)$ being the solute concentration at the outlet resulting from the simultaneous arrival of water particles which have experienced different travel times and have come into contact with different immobile phases concentrations. The distinction between resident and flux concentrations for non-steady advection is indeed well known (e.g. Rinaldo and Marani, 1987). Flux concentrations are needed, in particular, when considering serial transport volumes (see e.g. Eq. 29).

\section{Generalized applications}

In general, the determination of travel time distributions must be accomplished following an analysis of the detailed motion of water particles in space and time over a channel network. Indeed a complex catchment entails a nested structure of geomorphic states, quite different from one another, where hydrologic transport occurs. Typically one thinks of hillslopes (where solute generation within hydrologic runoff mostly occurs) and channel states (where usually routing occurs, though exchanges with hyporheic zones or riparian vegetation or biologic decays may be significant, especially if travel times therein become large). We thus need to define the collection $\Gamma$ of all individual paths $\gamma \in \Gamma$ that a particle may follow up to the basin outlet. The collection of connected paths $\gamma=x_{1}, x_{2}, \cdots x_{\Omega}$ (where we define $\Omega$ as the closure of the catchment) consists of the set of all feasible routes to the outlet, that is $x_{1} \rightarrow x_{2} \rightarrow \cdots \rightarrow x_{\Omega}$. A different notation clarifies the above geomorphic framework. If $A_{i}, i=1, N$ is the number of overland states whose total area covers the entire catchment (say, we neglect the actual surface of channelized patterns), and $c_{i}$ defines any channel link of the catchment ( $N$ is the total number of links), all the paths are supposed to originate within hillslopes i.e. $A_{i} \rightarrow c_{i} \rightarrow \cdots \rightarrow c_{\Omega}$, where $\Omega$ is the conventional notation for the outlet of the basin.

The above rules specify the spatial distribution of pathways available for hydrologic runoff through an arbitrary network of channel and overland regions. The travel time spent by a particle along any one of the above paths is composed by the sum of the residence times within each of the states actually composing the considered path. Nevertheless, the time $T_{x}$ that a particle spends in state $x \quad\left(x=A_{i}\right.$ or $x=c_{i}$ ) is a random variable which can be described by probability density functions (pdf's) $f_{x}(t)$. Obviously, for different states $x$ and $y, T_{x}$ and $T_{y}$ can have different pdf's $f_{x}(t) \neq f_{y}(t)$ and we assume that $T_{x}$ and $T_{y}$ are statistically independent for $x \neq y$. For a path $\gamma \in \Gamma$ defined by the collection of states $\gamma=\left\langle x_{1}, \ldots, x_{k}\right\rangle$ (where, in turn, $\left.x_{1}, \cdots, x_{k} \in\left(A_{1}, . ., A_{\Omega}, c_{1}, . ., c_{\Omega}\right)\right)$ we define a travel time $T_{\gamma}$ through the path $\gamma$ as:

$T_{\gamma}=T_{x_{1}}+\ldots \ldots+T_{x_{k}}$

From the statistical independence of the random variables $T_{x_{i}}$ it follows that the derived distribution $f_{\gamma}(t)$ of the sum of the (independent) residence times $T_{x_{i}}$ is the convolution of the individual pdf's:

$f_{\gamma}(t)=f_{x_{1}} * \cdots * f_{x_{k}}$

where the asterisk $*$ denotes the convolution operator.

Travel time distributions $f(t)$ at the outlet of a system whose input mass is distributed over the entire domain are obtained by randomization over all possible paths (Rodriguez-Iturbe and Valdes, 1979; Gupta et al., 1980):

$f(t)=\sum_{\gamma \in \Gamma} p(\gamma) f_{\gamma}(t)$ 
where $\gamma$ is the arbitrary path constituted of states $\left\langle x_{1}, \ldots, x_{k}\right\rangle$, $f_{\gamma}$ is the path travel time distribution as given by Eq. (21) and $\gamma$ is the arbitrary path from source to outlet; furthermore, $p(\gamma)$ is the path probability, i.e. $\sum_{\gamma \in \Gamma} p(\gamma)=1$, defining the relative proportion of particles in $\gamma$.

We now define (and generalize) different types of path probabilities. In the simplest case, the path probabilities may be simply defined as $p(\gamma)=A_{\gamma} / A$, where $A_{\gamma}$ is the contributing area draining into the first channel state of any given path $\gamma$. In such a case $\sum_{\gamma \in \Gamma} A_{\gamma}=A$, where $A$ is the total area drained by the channel network, and the path probability is solely determined by geomorphology. The above time-independent determination of the path probabilities is tantamount to assuming uniform rainfall in space, and this severely constrains the size of the catchment to be modeled, which is related to the basic scale of spatial heterogeneity of rainfall patterns.

Where rainfall patterns, say $j(\mathbf{x}, t)$, are distributed in space and time, the path probabilities would be simply dictated by the relative fraction of rainfall, i.e.

$p(\gamma, t)=\frac{\int_{A_{\gamma}} j(\mathbf{x}, t) d \mathbf{x}}{\int_{A} j(\mathbf{x}, t) d \mathbf{x}}=\frac{J(\gamma, t)}{J(t)}$

(where $J(\gamma, t) d t=d t \int_{A_{\gamma}} j(\mathbf{x}, t) d \mathbf{x}$ is the total quantity of rainfall entering the system in $(t-d t, t)$ through the path $\gamma$, and $J(t) d t$ the total rainfall injected in the same period over the entire watershed) which allows to embed any rainfall pattern in space and time routing them through the catchment at each time interval. This capability is central to the innovation contained in our model, and constitutes a new and relevant extension of traditional GIUH approaches.

Whether a pattern in space and time of $j(\mathbf{x}, t)$ derives from the characters of rainfall or of runoff production will be seen elsewhere. Notice that we may derive arbitrary rainfall fields either by kriging of point rainfall measurements, or by assuming stochastic patterns derived from theoretical models. Hence one might derive the rainfall-weighted path probabilities in the general case by simple quadratures. A reliable operational procedure consists of isolating through suitable drainage directions on digital terrain maps a spanning set of subbasins of size considerably smaller than the macroscales of intense rainfall patterns, thereby defining spanning sets of landing areas $\gamma$ where one can assume locally constant rainfall intensity $J(\gamma, t)$. This procedure is tantamount to a coarse-graining of the original rainfall patterns from the pixel size to that of a collection of thousands of them, with much improved computational efficiency at no cost of predictive loss. Moreover, any spatially distributed model of runoff production would result in distributions of input $j(\mathbf{x}, t)$ more markedly heterogeneous in space.

Moreover, whether or not one needs to modify travel times depending on the intensity of the hydrologic events (e.g. geomorphoclimatically) depends by the modes of hydrologic transport, say when dominated by storage rather than kine- matic effects, but the basic formal machinery remains unaffected. Many papers have addressed the characterization of travel times and the related hydrologic response. We will not review them here. Suffice here to say that the description of hillslope transport is of great importance (e.g. Rinaldo et al., 1995; Robinson and Sivapalan, 1995; Botter and Rinaldo, 2003). In fact, hillslope residence times are responsible not only for key lags (and rather complex mechanisms like preferential pathways to runoff) in the overall routing, but are also important to the understanding of derived transport processes, chiefly solute generation and transport to runoff waters. The above matter, jointly with the physical problem of characterizing well where channels begin, still needs to be resolved satisfactorily.

In the framework previously depicted, flowrates are obtained by propagating spatially distributed, time-dependent net rainfall impulses by the use of linear invariant hydrologic responses. The basic formulation of the geomorphologic theory of the hydrologic response is thus given by the following convolution integral:

$Q_{w}(t)=\int_{0}^{t} d t_{0} J\left(t_{0}\right) \sum_{\gamma \in \Gamma} p\left(\gamma, t_{0}\right) f_{\gamma}\left(t-t_{0}\right)$

In the occurrence of spatially uniform, time varying net rainfall intensity $J(t)$ one has

$$
\begin{aligned}
Q_{w}(t) & =\int_{0}^{t} d t_{0} J\left(t_{0}\right) \sum_{\gamma \in \Gamma} p(\gamma) f_{\gamma}\left(t-t_{0}\right) \\
& =\int_{0}^{t} d t_{0} J\left(t_{0}\right) f\left(t-t_{0}\right)
\end{aligned}
$$

because $f(t)=\sum_{\gamma \in \Gamma} p(\gamma) f_{\gamma}(t)$, and we recover the usual GIUH relationship (Gupta et al., 1980) which is employed in several practical cases. It should be stressed that the general formulation of Eq. (24) uses rainfall patterns in space and time both for determining the path probabilities $p(\gamma, t)$ and for filtering the net contribution $J(t)$.

The convolution integrals up to Eqs. (24) and (25) may be solved exactly for a number of cases (Rinaldo et al., 1991) where the dynamical parameters determining the propagation of the flood wave are assumed to be uniform. Alternatively, we may allow arbitrary variations in celerity and hydrodynamic dispersion, and thus numerical convolutions are often in order. In such cases, arbitrary travel time distributions may be used depending on the hydraulics and suitable numerical techniques (typically employing integral transforms) are used to accurately convolute in time. A strong control over the numerical machinery is obviously provided by continuity, given that $\int_{0}^{\infty} f_{\gamma}(\tau) d \tau \equiv 1 \forall \gamma$.

We note that the key identification of the paths $\gamma \in \Gamma$ may be done directly from digital terrain maps, hence exploiting our capabilities of extracting useful geomorphic information from them and chiefly the extent of the channelized portion of the basin (see e.g. Rodriguez-Iturbe and Rinaldo, 1997). 
From the results of the previous section, solute mass discharge is given in the following form:

$$
Q_{s}(t)=\int_{0}^{t} d t_{0} J\left(t_{0}\right) \sum_{\gamma \in \Gamma} p\left(\gamma, t_{0}\right) C_{\gamma}\left(t-t_{0}, t_{0}\right) f_{\gamma}\left(t-t_{0}\right)
$$

where $C_{\gamma}$ is a "path" resident concentration. In the case of water flow one simply has $C_{\gamma}=\rho$, the density of water. In this case $Q_{s}(t) / \rho$ becomes a flowrate, $Q_{w}\left[L^{3} / T\right]$, and Eqs. (24) and (25) are straightforwardly recovered.

The particular formulation of a mass-response function (MRF) approach depends on the number and the arrangement of the reacting states. A (relatively) simple case is that of a path (say $\gamma=x_{1} \rightarrow \ldots \rightarrow x_{\Omega}$, where $x_{\Omega}$ denotes, as usual, the terminal reach of the catchment), where the state $x_{1}$ generates solute mass to the mobile phase (hence one has mobile and immobile concentrations in $x_{1}$ denoted by $\left.C_{x_{1}}(t, \tau), N_{x_{1}}(t)\right)$, and all other states (from $x_{2}$ to $x_{\Omega}$ ) route the transported matter without further exchanges. In this case one has in Eq. (26):

$C_{\gamma}(t, 0) f_{\gamma}(t)=f_{x_{1}} C_{x_{1}}(t, 0) * f_{x_{2}} * \cdots * f_{x_{\omega}}$

In the general case where $x_{1}$ is a "generation" state (wherein solutes are transferred from the immobile to the mobile phase) and $x_{2}, x_{3}, \ldots, x_{\Omega}$ are reactive states where the solutes transported by the carrier may be retarded owing to chemical processes occurring with other immobile phases (e.g. bed sediment or dead zones that define chemical, biological or physical reactions), the mass response function may be expressed as:

$C_{\gamma}(t, 0) f_{\gamma}(t)=f_{x_{1}} C_{x_{1}}(t, 0) * f_{x_{2}} \lambda_{x_{2}} * \cdots * f_{x_{\Omega}} \lambda_{x_{\Omega}}$

where $\lambda_{x_{i}}(i=2, k)$ represents the gain/loss function within each reactive state forced by a non-null input flux concentration of solute $C_{x_{i}}^{F, \text { in }}(t) \neq 0$ :

$\lambda_{x_{i}}\left(t-t_{0}, t_{0}\right)=\frac{C_{x_{i}}\left(t-t_{0}, t_{0}\right)}{C_{x_{i}}^{F, i n}\left(t_{0}\right)}$

Obviously when downstream states route the matter without sorption we have $\lambda_{x_{i}} \equiv 1$. The notation $C_{x_{i}}$ and $\lambda_{x_{i}}$ should not surprise, as we argued that for each state where gain/loss processes occur one needs to carry out a global mass balance to determine the instantaneous fraction of matter stored in immobile phases $N_{x_{i}}(t)$. We argue that Eq. (28) is the general form of Mass Response Function (MRF) which, in different forms that reduce to particular cases of Eq. (28), has been known for some time (see e.g. Rinaldo and Marani, 1987).

On this basis alone one needs to weigh carefully the spatial and temporal scales relevant to a mathematical model of transport at catchment scales. All possible combinations of states generating, losing or simply routing solutes may thus be explored, thus extending the geomorphic theory of the hydrologic response to solute transport.

\section{Discussion}

The linkage of travel times with the global, basin-scale contact times between phases controlling mass exchanges provides a quantum leap in our operational capabilities of describing large-scale transport processes. Indeed a complex catchment entails a nested structure of geomorphic states where the spatial pathways of any rain-driven particle moving through the network of channel and overland regions define the control volumes for which one needs to carry out mass balances and compute travel and lifetime distributions.

We shall discuss a few examples with the scope of clarifying the structure of mass response functions. The examples are kept to a minimum of geomorphic and hydrologic complexity to avoid clouding the main issue. Rainfall is assumed constant in space, i.e. $p(\gamma, t)=p(\gamma)$. Figure 1 shows the chosen setup, composed of five source areas and five channels. Overall, the topological order is $\Omega=2$.

The complete set $\Gamma$ of paths to the outlet (see Fig. 1) is the following:

$$
\begin{aligned}
& A_{1} \rightarrow c_{1} \rightarrow c_{3} \rightarrow c_{5} \\
& A_{2} \rightarrow c_{2} \rightarrow c_{3} \rightarrow c_{5} \\
& A_{3} \rightarrow c_{3} \rightarrow c_{5} \\
& A_{4} \rightarrow c_{4} \rightarrow c_{5} \\
& A_{5} \rightarrow c_{5}
\end{aligned}
$$

The states where paths originate are labeled by an area $A_{i}$, so that the total catchment area $A$ obeys the relation $A=A_{1}+\cdots+A_{5}$ and path probabilities are defined by $p(1)=A_{1} / A ; \ldots ; p(5)=A_{5} / A$, thereby assuming that the rainfall is spatially uniform - this is tantamount to assuming that the watershed "width" is smaller than the correlation scale of rainfall events. Under the circumstances shown in Fig. 1, Eq. (22) applies with:

$$
\begin{aligned}
f(t)= & \frac{A_{1}}{A} f_{A_{1}} * f_{c_{1}} * f_{c_{3}} * f_{c_{5}}+ \\
& \frac{A_{2}}{A} f_{A_{2}} * f_{c_{2}} * f_{c_{3}} * f_{c_{5}}+\frac{A_{3}}{A} f_{A_{3}} * f_{c_{3}} * f_{c_{5}}+ \\
& +\frac{A_{4}}{A} f_{A_{4}} * f_{c_{4}} * f_{c_{5}}+\frac{A_{5}}{A} f_{A_{5}} * f_{c_{5}}
\end{aligned}
$$

where we have neglected for the sake of simplicity the probability for a particle to land directly on a channel state).

Note that the transition $A_{i} \rightarrow c_{i}$ (i.e. hillslope to channel) entails a subtle point modelling issue, in fact, here we assume to describe the overall travel time distribution by a convolution of $f_{A_{i}}(t)$ and $f_{c_{i}}(t)$, where $f_{A_{i}}(t)$ is the hillslope travel time distribution, regardless of the point where the channel is reached, and $f_{c_{i}}(t)$ is the travel time distribution computed for the total length of the channel. In reality one should take into account the actual distribution of injections along the entire channel reach, rather than a fictitious headwater injection. The issue of the equivalence of the results has been studied by a number of authors (for a review see Rinaldo 


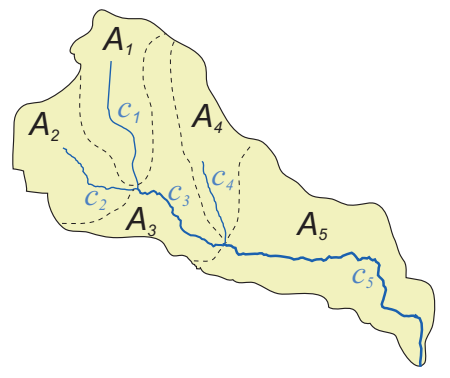

(a)

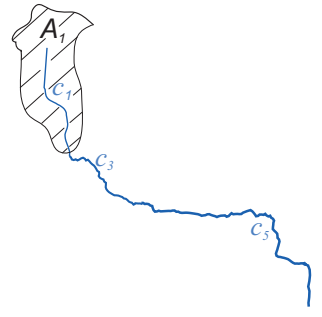

(b)
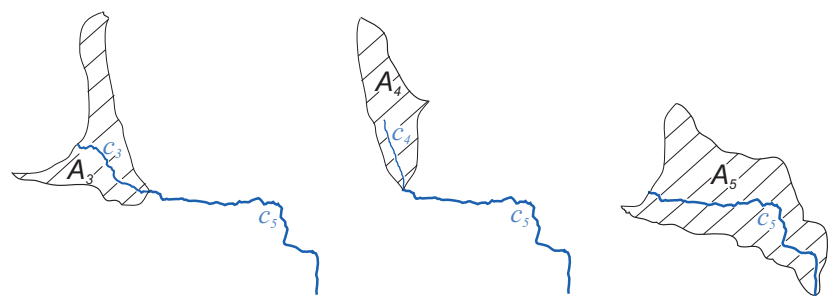

Fig. 1. (a) Parallel transport. Sample of a relatively simple geomorphological structure of a river basin and notation for the theoretical models. The basic elements of the MRF approach for basin scale solute transport are provided. Notice that the set $\Gamma$ of all possible paths to the outlet defined by the geomorphic structure is made up by 10 states, five overland states and five channels (e.g. transitions to overland areas $A_{i}$ to their outlet channel $c_{i}$ and then to ensuing transitions $\left(c_{i} \rightarrow c_{k} \rightarrow \cdots \rightarrow c_{5}\right)$ towards the closure - the endpoint of channel $c_{5}$ ). Notice the treatment of the $i$-th source area $A_{i}$ as a well-mixed reactor. Here we assume that all sources areas $A_{1}$ to $A_{5}$ act as generators of solutes to the mobile phase emphasizing their independent role possibly related to land use; (b) The set of independent paths available for hydrologic runoff is enumerated and shown.

and Rodriguez-Iturbe, 1996), and here we simply claim that our scheme represents a reasonable approximation in view, in particular, of other assumptions involved.

Figure 2a shows the individual travel time distributions for the path $\gamma_{1}$ defined by the transitions: $A_{1} \rightarrow c_{1} \rightarrow c_{3} \rightarrow c_{5}$. Also shown (Fig. 2b) is a comparison of the path, $f_{\gamma_{(1)}}$, and the basin, $f(t)$, travel time distributions needed for the general definition of fluxes. The comparison shows the obvious blending of different arrivals that reflect the geomorphological complexity of the pathways to the outlet.

Mass response functions are easily determined when parallel generation states occur. If we assume that every hillslope $A_{i}$ acts as a generator of solute matter to runoff (a usual
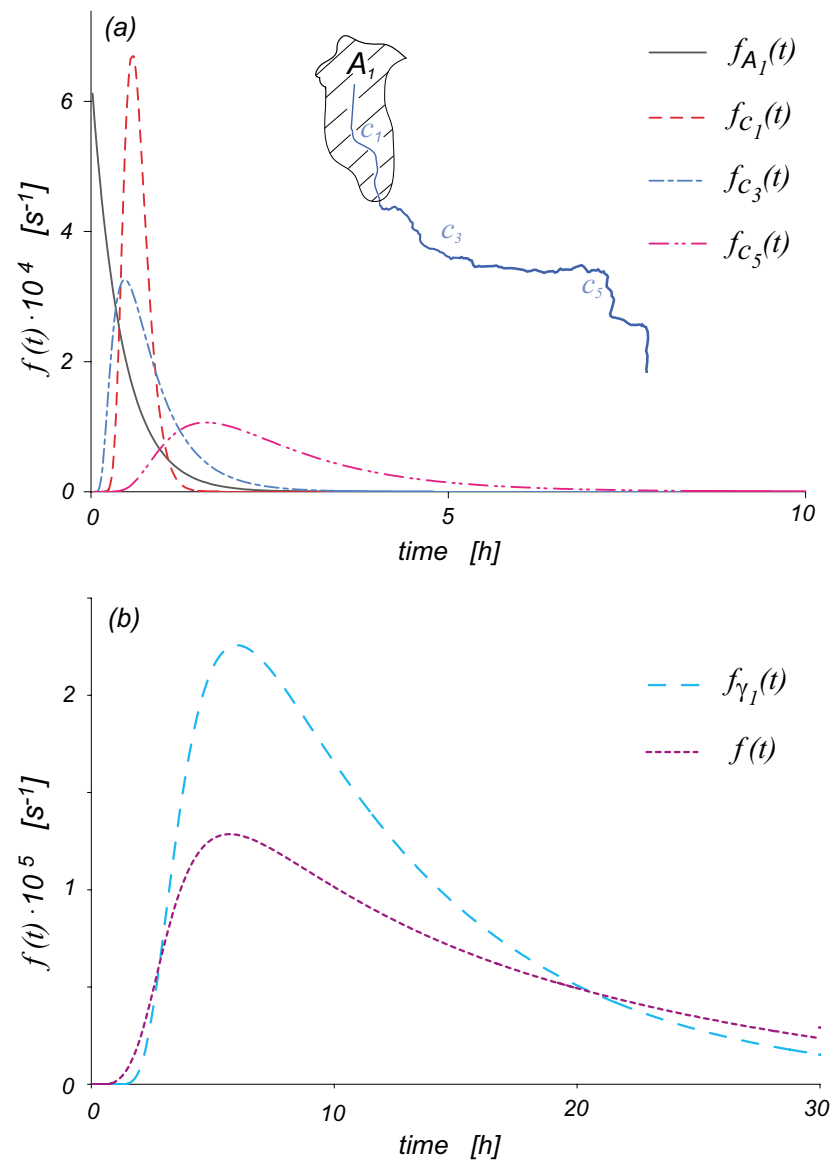

Fig. 2. (a) Individual travel time distributions along the path $A_{1} \rightarrow \cdots \rightarrow c_{5}$; (b) Travel time distribution $f_{\gamma_{1}}(t)$ obtained by convolution of the individual pdfs, and catchment travel time distribution $f(t)$.

assumption in nonpoint source pollution studies), we have, for the water pulse injected at $t_{0}=0$ (i.e. $\tau=t$ ) is:

$$
\begin{gathered}
\sum_{\gamma} p(\gamma) C_{\gamma}(t, 0) f_{\gamma}(t)=\frac{A_{1}}{A} f_{A_{1}} C_{A_{1}}(t, 0) * f_{c_{1}} * f_{c_{3}} * f_{c_{5}}+ \\
+\frac{A_{2}}{A} f_{A_{2}} C_{A_{2}}(t, 0) * f_{c_{2}} * f_{c_{3}} * f_{c_{5}}+\frac{A_{3}}{A} f_{A_{3}} C_{A_{3}}(t, 0) * f_{c_{3}} * f_{c_{5}} \\
+\frac{A_{4}}{A} f_{A_{4}} C_{A_{4}}(t, 0) * f_{c_{4}} * f_{c_{5}}+\frac{A_{5}}{A} f_{A_{5}} C_{A_{5}}(t, 0) * f_{c_{5}}
\end{gathered}
$$

which defines the mass-response function for the basin shown in Fig. 1. Note that for a unit pulse of rainfall one has $Q_{s}(t)=\sum_{\gamma} p(\gamma) C_{\gamma}(t, 0) f_{\gamma}(t)$, while for compounded inputs of rainfall $J(t)$ one has to solve Eq. (26).

Examples of computations are shown in Fig. 3, where results for an instantaneous unit pulse of effective rainfall $J(t)=\delta(t)$ are reported in panels (a) and (b). Figure 3a shows the connected behavior of the resident mobile, $C$, and immobile, $N$, concentrations in state $A_{1}$ obtained by solving 

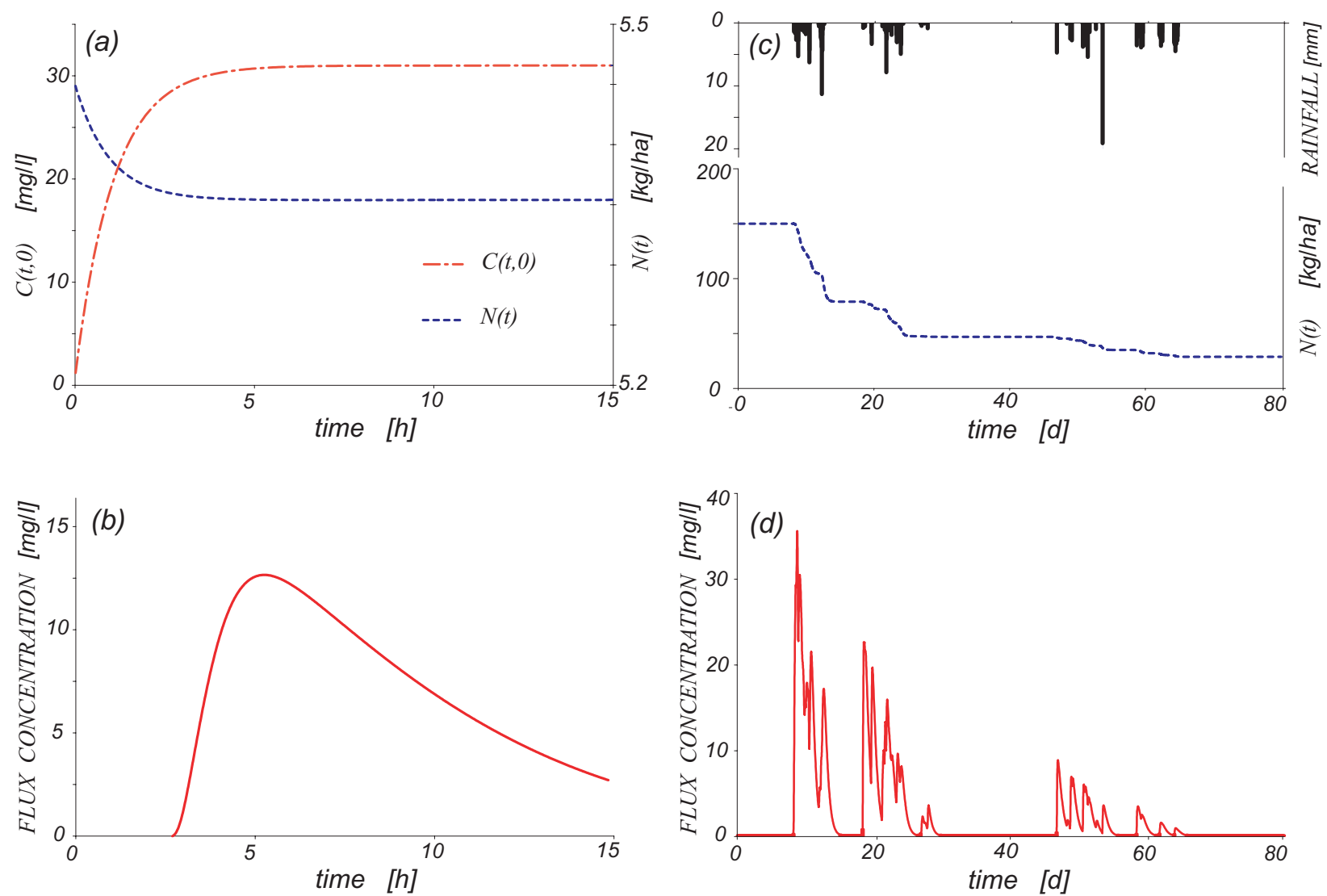

Fig. 3. Parallel transport: (a) Resident concentration $C_{A_{1}}(t, 0)$ and its corresponding immobile phase concentration $N_{A_{1}}(t)$ (expressed in $\mathrm{kg} / \mathrm{ha}$ ) vs. $t$ for an instantaneous pulse; (b) flux concentration at the outlet of the basin $C^{F}{ }_{c_{5}}(t)$; (c) temporal evolution of the rainfall depths (upper plot) and corresponding immobile phase concentration $N_{A_{1}}(t)$ for a sequence of intermittent rainfall pulses, a case typical of transport in the hydrologic runoff. Also shown in (d) is the corresponding flux concentration at the outlet of the catchment, $C^{F}{ }_{c_{5}}(t)$.

Eq. (12) with a given initial concentration $N(0)$ and initially zero concentration in mobile phase $C(0,0)=0$. Note that the particular choice of numerical value of $N(0)$ (here about $5.45[\mathrm{~kg} / \mathrm{ha}])$ is immaterial. The flux concentration at the outlet is obtained by solving five mass balance equations of the type (Eq. 12) for the five generating states $A_{i}$ to determine five different path concentrations $C_{\gamma}(\tau, t, 0)$, and then posing $Q_{s}(t)=\sum_{\gamma} p(\gamma) C_{\gamma}(t, 0) f_{\gamma}(t)$ and $C^{F}(t)=Q_{s} / Q_{w}$, which is the final result shown in Fig. 3b. Figure $3 \mathrm{c}$, instead, describes a case where a sequence of rainfall inputs $J(t)$ (shown in the upper plot) drives a complex chain of events, thus requiring more complex computations. In the lower plot of Fig. 3c we show the behavior of $N(t)$ in one of the generating states, evidencing the effect of solute leaching due to the sequence of rainfall impulses. One may also notice the reduced rates of solute generation to runoff for the late-coming pulses (most of the mass had been leached previously), which reflect the lack of translational invariance postulated by the dependence of resident concentrations onto two different timescales, i.e. $C=C\left(\tau, t_{0}\right)$. The plot reported in Fig. 3d has been obtained by solving Eq. (26) with the sequence of $J(t)$ reported in Fig. 3c, in the case of parallel generation and transport of solutes.

A second example, involving serial transport, is more complex. If we assume that mass loss/gain processes are significant in serial states (two hillslopes and a stream channel, see Fig. 4), one may specifically assume that: i) the overland states $A_{1}$ and $A_{4}$ are generation states, like e.g. agricultural areas where fertilization occurs; ii) the stream channel $c_{5}$ is a relatively vegetated, high-residence time channel reach where reaction processes matter. In this case the travel time distributions is the same of the case above, whereas the MRF for the water pulse injected at $t_{0}=0(\tau=t)$ :

$$
\begin{aligned}
& \sum_{\gamma} p(\gamma) C_{\gamma}(t, 0) f_{\gamma}(t)= \\
= & \frac{A_{1}}{A} f_{A_{1}} C_{A_{1}}(t, 0) * f_{c_{1}} * f_{c_{3}} * f_{c_{5}} \frac{C_{c_{5}}}{C_{c_{5}}^{F, i n}} \\
+ & \frac{A_{4}}{A} f_{A_{4}} C_{A_{4}}(t, 0) * f_{c_{4}} * f_{c_{5}} \frac{C_{c_{5}}}{C_{c_{5}}^{F, i n}}
\end{aligned}
$$



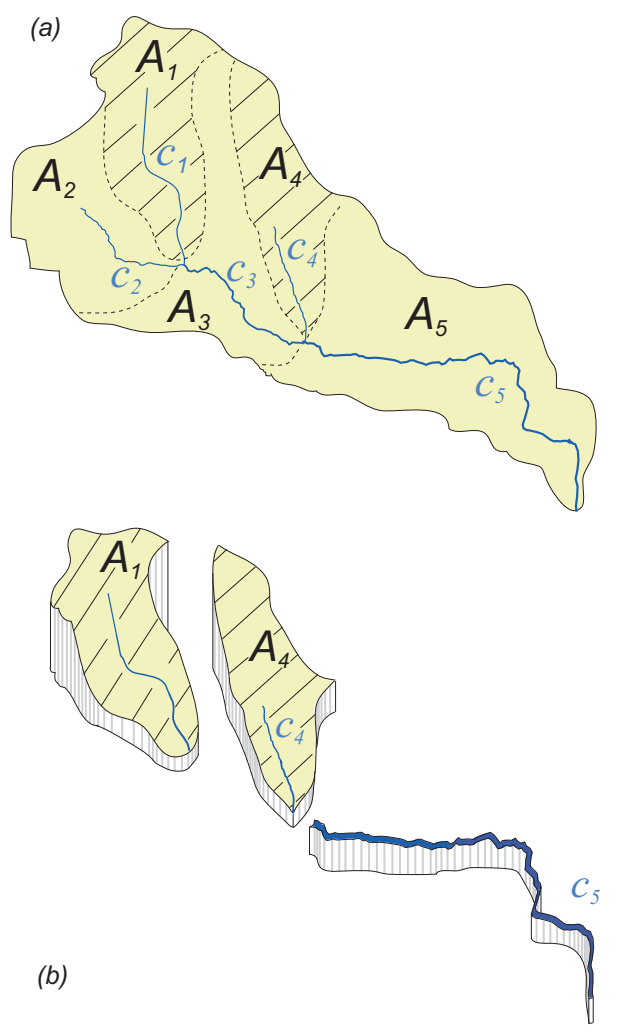

Fig. 4. Serial transport: (a) geomorphological structure of the test catchment: here we assume that only sources areas $A_{1}$ and $A_{4}$ act as generators of solutes to the mobile phase. Moreover, we assume that mass transfer processes also occur in state $c_{5}$ owing to its travel times and nature. Reactive states $\left(A_{1}, A_{4}\right.$ and $\left.c_{5}\right)$ are properly isolated in (b).

where the resident concentrations in the reactive state that follows the solute generation (i.e. channel 5) is properly normalized by the inflowing flux concentration. Note that only the contributions of "source" states explicitly appear in the MRF, whereas large dilutions determined by all the states generating clean runoff are reflected by lower flux concentration along the stream network. Needless to say, the serial arrangement is considerably more involved computationally.

Every possible combination is thus tackled, and a suitable extension of the geomorphic theory of the hydrologic response to transport at basin scales is therefore achieved.

Acknowledgements. This research is funded by EU project AQUATERRA (GOCE 505428). G. Botter and M. Marani acknowledge support of CORILA (Consorzio per la Gestione del Centro di Coordinamento delle attività di Ricerca inerenti il Sistema Lagunare) through its grant 3.17/2004.

Edited by: P. Grathwohl

\section{References}

Beven, K. and Freer, J.: Equality, data assimilation and uncertainty estimation in mechanistic modelling of complex environmental systems using the GLUE methodology, J. Hydrol., 249, 1-4, 2001.

Botter, G. and Rinaldo, A.: Scale effects on geomorphologic and kinematic dispersion, Water Resour. Res., 39, 1286-1294, 2003.

Botter, G., Bertuzzo, E., Bellin, A., and Rinaldo, A.: On the Lagrangian formulations of reactive solute transport in the hydrologic response, Water Resour. Res., 41, 4008-4016, 2005.

Cvetkovic, V. and Dagan, G.: Transport of kinetically sorbing solute by steady random velocity in heterogeneous porous formations, J. Fluid Mech., 265, 189-215, 1994.

Cvetkovic, V. and Dagan, G.: Reactive transport and immiscible flow in geological media. I. General theory, Proc. R. Soc. Lond., A452, 303-328, 1996.

Dagan G.: Flow and transport in porous formations, SpringerVerlag, Berlin-Heidelberg, 1989.

Destouni, G. and Graham, W. D.: Solute Transport Through an Integrated Heterogeneous Soil-Groundwater system, Water Resour. Res., 31, 1935-1944, 1995.

Gupta, V. K., Waymire, E., and Wang, C. T.: A representation of an IUH from geomorphology, Water Resour. Res., 16, 885-862, 1980.

Gupta, A. and Cvetkovic, V.: Material transport from different sources in a network of streams through a catchment, Water Resour. Res., 38, 1098-2004, 2002.

Maurer, E. P. and Lettenmaier, D. P.: Predictability of seasonal runoff in the Mississippi River basin, J. Geophys. Res.Atmosphere, 108, 8607-8615, 2003.

Montanari, A. and Uhlenbrook, S.: Catchment modeling: towards an improved representation of the hydrological processes in realworld model application, J. Hydrol., 291, 159-160, 2004.

Rinaldo, A. and Marani, A.: Basin scale model of solute transport, Water Resour. Res., 23, 2107-2118, 1987.

Rinaldo, A., Bellin, A., and Marani, A.: On mass response functions, Water Resour. Res., 25, 1603-1617, 1989.

Rinaldo, A., Rigon, R., and Marani, A.: Geomorphologic dispersion, Water Resour. Res., 28, 513-525, 1991.

Rinaldo, A., Dietrich, W. E., Rigon, R., Vogel, G. K., and Rodriguez-Iturbe, I.: Can one gauge the shape of a basin?, Water Resour. Res., 31, 1119-1127, 1995.

Rinaldo, A. and Rodriguez-Iturbe, I.: Geomorphological theory of the hydrologic response, Hydrol. Processes, 10, 803-844, 1996.

Rinaldo, A., Bertuzzo, E., and Botter, G.: Nonpoint source transport models from empiricism to coherent theoretical frameworks, Ecological modeling, 184, 19-35, 2005.

Robinson, J. S., Sivapalan, M., and Snell, J. D.: On the relative roles of hillsolpe process, channel routing, and network morphology in the hydrologic response of natural catchments, Water Resour. Res., 30, 3089-3101, 1995.

Rodriguez-Iturbe, I. and Valdes, J. B.: The geomorphologic structure of hydrologic response, Water Resour. Res., 15, 1409-1420, 1979.

Rodriguez-Iturbe, I. and Rinaldo, A.: Fractal River Basins. Chance and Self-Organization, Cambridge University Press, New York, 1997.

Simic, E. and Destouni, G.: Water and solute residence times in a catchment: Stochastic-mechanistic model interpretation of 
A. Rinaldo et al.: Basin-scale transport: 1 .

oxygen-18 transport, Water Resour. Res., 35, 2109-2119, 1999.

Taylor, G. I.: Diffusion by continuous movements, Proc. London Math. Soc. Ser., A 20, 196-211, 1921.
Van Genuchten, M. T.: Analytical solution for chemical transport with simultaneous adsorption, zero-order production and firstorder decay, J. Hydrol., 49, 213-233, 1981. 